\title{
Vitamin D and COVID-19 infection and mortality in UK Biobank
}

\author{
Claire E. Hastie ${ }^{1}$ (D) Jill P. Pell ${ }^{1}$ - Naveed Sattar ${ }^{2}$ (D)
}

Received: 29 June 2020 / Accepted: 19 August 2020 / Published online: 26 August 2020

(c) The Author(s) 2020

\begin{abstract}
Purpose Low blood 25-hydroxyvitamin D (25(OH)D) concentration has been proposed as a potential causal factor in COVID-19 risk. We aimed to establish whether baseline serum 25(OH)D concentration was associated with COVID-19 mortality, and inpatient confirmed COVID-19 infection, in UK Biobank participants.

Methods UK Biobank recruited 502,624 participants aged 37-73 years between 2006 and 2010. Baseline exposure data, including serum 25(OH)D concentration, were linked to COVID-19 mortality. Univariable and multivariable Cox proportional hazards regression analyses were performed for the association between 25(OH)D and COVID-19 death, and Poisson regression analyses for the association between 25(OH)D and severe COVID-19 infection.

Results Complete data were available for 341,484 UK Biobank participants, of which 656 had inpatient confirmed COVID-19 infection and 203 died of COVID-19 infection. 25(OH)D concentration was associated with severe COVID-19 infection and mortality univariably (mortality per $10 \mathrm{nmol} / \mathrm{L} 25(\mathrm{OH}) \mathrm{D}$ HR $0.92 ; 95 \% \mathrm{CI} 0.86-0.98 ; p=0.016$ ), but not after adjustment for confounders (mortality per $10 \mathrm{nmol} / \mathrm{L} 25(\mathrm{OH}) \mathrm{D}$ HR $0.98 ; 95 \% \mathrm{CI}=0.91-1.06 ; p=0.696$ ). Vitamin D insufficiency or deficiency was also not independently associated with either COVID-19 infection or linked mortality.

Conclusions Our findings do not support a potential link between 25(OH)D concentrations and risk of severe COVID-19 infection and mortality. Randomised trials are needed to prove a beneficial role for vitamin $\mathrm{D}$ in the prevention of severe COVID-19 reactions or death.
\end{abstract}

Keywords COVID-19 $\cdot$ Vitamin D · Mortality

In the hunt for modifiable COVID-19 risk factors, vitamin $D$ has gained a lot of attention both in the media and within the scientific community [1]. Proponents of such a link cite a few early studies that present circumstantial evidence. They are either ecological, at an individual level but unable to fully adjust for potential confounders, or they measured 25-hydroxyvitamin D (25(OH)D) concentration once patients were already hospitalised with COVID-19 which introduces reverse causation, as vitamin D is a negative acute phase reactant [2].

Jill P. Pell and Naveed Sattar are joint senior authors.

Claire E. Hastie

claire.hastie@glasgow.ac.uk

1 Institute of Health and Wellbeing, University of Glasgow, 1 Lilybank Gardens, Glasgow G12 8RZ, UK

2 Institute of Cardiovascular and Medical Sciences, University of Glasgow, Glasgow, UK
Despite the sparse evidence on vitamin D in COVID-19 [3], the UK government led an urgent review into whether there is any link. It concluded that "There is no evidence to support taking vitamin $\mathrm{D}$ supplements to specifically prevent or treat COVID-19" [4]. By contrast, the Welsh COVID-19 risk assessment tool includes vitamin D supplementation as part of its recommendations [5]. Furthermore, a recent review conducted by the Scientific Advisory Committee on Nutrition found insufficient evidence to support recommending vitamin D supplementation to prevent acute respiratory tract infections in the general UK population [6].

We previously observed no evidence of an association between baseline serum $25(\mathrm{OH}) \mathrm{D}$ concentration and testing positive for SARS-CoV-2 in hospital in UK Biobank once potential confounders were adjusted for [7]. Importantly, some of the variables that were associated with increased COVID-19 risk in our sample, for example lower socioeconomic status, being Black or South Asian, or being overweight or obese, are also associated with lower vitamin D. This suggests that the positive findings of other studies may 
Table 1 Univariable association between baseline covariates and confirmed COVID-19 mortality, and confirmed inpatient COVID-19 infection

\begin{tabular}{|c|c|c|c|c|}
\hline & \multicolumn{2}{|c|}{ COVID-19 mortality } & \multicolumn{2}{|c|}{ Inpatient COVID-19 infection } \\
\hline & HR $(95 \% \mathrm{CI})$ & $p$ & $\operatorname{IRR}(95 \% \mathrm{CI})$ & $p$ \\
\hline \multicolumn{5}{|l|}{ Sex } \\
\hline Female & 1 & & 1 & \\
\hline Male & $2.49(1.85-3.35)$ & $<0.001$ & $1.50(1.29-1.75)$ & $<0.001$ \\
\hline \multicolumn{5}{|l|}{ Self-reported ethnicity } \\
\hline White & 1 & & 1 & \\
\hline Black & $7.44(4.30-12.9)$ & $<0.001$ & $3.14(2.17-4.53)$ & $<0.001$ \\
\hline South Asian & $1.76(0.65-4.74)$ & 0.264 & $2.69(1.87-3.89)$ & $<0.001$ \\
\hline \multicolumn{5}{|c|}{ Townsend deprivation quintile } \\
\hline 1 & 1 & & 1 & \\
\hline 2 & $1.05(0.64-1.72)$ & 0.855 & $1.04(0.79-1.36)$ & 0.802 \\
\hline 3 & $0.95(0.57-1.58)$ & 0.845 & $1.08(0.82-1.42)$ & 0.584 \\
\hline 4 & $1.47(0.93-2.34)$ & 0.101 & $1.37(1.06-1.77)$ & 0.016 \\
\hline 5 & $2.56(1.67-3.91)$ & $<0.001$ & $2.23(1.75-2.83)$ & $<0.001$ \\
\hline \multicolumn{5}{|l|}{ Household income } \\
\hline$<£ 18,000$ & 1 & & 1 & \\
\hline$£ 18 \mathrm{k}-£ 30,999$ & $0.47(0.33-0.67)$ & $<0.001$ & $0.62(0.51-0.76)$ & $<0.001$ \\
\hline$£ 31 \mathrm{k}-£ 51,999$ & $0.42(0.29-0.60)$ & $<0.001$ & $0.55(0.45-0.68)$ & $<0.001$ \\
\hline$£ 52 \mathrm{k}-£ 100,000$ & $0.28(0.18-0.44)$ & $<0.001$ & $0.44(0.34-0.55)$ & $<0.001$ \\
\hline$>£ 100,000$ & $0.14(0.04-0.43)$ & 0.001 & $0.36(0.23-0.56)$ & $<0.001$ \\
\hline \multicolumn{5}{|l|}{ Overall health rating } \\
\hline Excellent & 1 & & 1 & \\
\hline Good & $1.52(0.94-2.43)$ & 0.085 & $1.49(1.15-1.93)$ & 0.003 \\
\hline Fair & $3.49(2.14-5.68)$ & $<0.001$ & $2.46(1.86-3.26)$ & $<0.001$ \\
\hline Poor & $4.86(2.59-9.12)$ & $<0.001$ & $4.97(3.54-6.96)$ & $<0.001$ \\
\hline \multicolumn{5}{|c|}{ Long-standing illness, disability or infirmity } \\
\hline No & 1 & & 1 & \\
\hline Yes & $2.80(2.13-3.70)$ & $<0.001$ & $1.84(1.58-2.15)$ & $<0.001$ \\
\hline \multicolumn{5}{|l|}{ Smoking status } \\
\hline No & 1 & & 1 & \\
\hline Yes & $1.36(0.91-2.02)$ & 0.134 & $1.34(1.07-1.69)$ & 0.013 \\
\hline \multicolumn{5}{|l|}{ BMI category } \\
\hline Normal weight & 1 & & 1 & \\
\hline Underweight & $1.86(0.26-13.6)$ & 0.539 & $1.38(0.44-4.31)$ & 0.584 \\
\hline Overweight & $1.82(1.23-2.67)$ & 0.002 & $1.50(1.23-1.83)$ & $<0.001$ \\
\hline Obese & $3.13(2.12-4.62)$ & $<0.001$ & $2.02(1.64-2.49)$ & $<0.001$ \\
\hline \multicolumn{5}{|l|}{ Diabetes } \\
\hline No & 1 & & 1 & \\
\hline Yes & $5.06(3.59-7.13)$ & $<0.001$ & $2.49(1.96-3.17)$ & $<0.001$ \\
\hline Current age (years) & $1.13(1.11-1.16)$ & $<0.001$ & $1.01(1.00-1.02)$ & 0.007 \\
\hline $\begin{array}{l}\text { Baseline systolic blood } \\
\text { pressure }(\mathrm{mmHg})\end{array}$ & $1.02(1.02-1.03)$ & $<0.001$ & $1.00(0.999-1.01)$ & 0.123 \\
\hline $\begin{array}{l}\text { Baseline diastolic } \\
\text { blood pressure } \\
(\mathrm{mmHg})\end{array}$ & $1.01(0.998-1.03)$ & 0.099 & $1.01(0.999-1.01)$ & 0.109 \\
\hline
\end{tabular}

$H R$ hazard ratio, $C I$ confidence interval, IRR incidence rate ratio in part be due to inadequate adjustment. Another recent study of UK Biobank data replicated this finding [8], but it would be more informative to relate $25(\mathrm{OH}) \mathrm{D}$ concentration to COVID-19-related mortality. 
Table 2 Association between baseline serum 25(OH)D and confirmed COVID-19 mortality, and confirmed inpatient COVID-19 infection

\begin{tabular}{|c|c|c|c|c|c|c|c|c|}
\hline & \multicolumn{4}{|c|}{ COVID-19 mortality } & \multicolumn{4}{|c|}{ Inpatient COVID-19 infection } \\
\hline & \multicolumn{2}{|l|}{ Univariable } & \multicolumn{2}{|l|}{ Multivariable* } & \multicolumn{2}{|l|}{ Univariable } & \multicolumn{2}{|l|}{ Multivariable* } \\
\hline & $\mathrm{HR}(95 \% \mathrm{CI})$ & $p$ value & $\mathrm{HR}(95 \% \mathrm{CI})$ & $p$ value & IRR (95\% CI) & $p$ value & $\overline{\text { IRR }(95 \% \mathrm{CI})}$ & $p$ value \\
\hline 25(OH)D (per $10 \mathrm{nmol} / \mathrm{L})$ & $0.92(0.86-0.98)$ & 0.016 & $0.98(0.91-1.06)$ & 0.696 & $0.93(0.90-0.97)$ & $<0.001$ & $1.00(0.96-1.05)$ & 0.888 \\
\hline $\begin{array}{l}\text { Vitamin D deficient }(25(\mathrm{OH}) \\
\mathrm{D}<25 \mathrm{nmol} / \mathrm{L})\end{array}$ & $1.61(1.14-2.27)$ & 0.007 & $1.21(0.83-1.76)$ & 0.311 & $1.56(1.28-1.90)$ & $<0.001$ & $1.10(0.88-1.37)$ & 0.404 \\
\hline $\begin{array}{l}\text { Vitamin D insufficient }(25(\mathrm{OH}) \\
\mathrm{D}<50 \mathrm{nmol} / \mathrm{L})\end{array}$ & $1.29(0.97-1.72)$ & 0.076 & $1.02(0.75-1.38)$ & 0.919 & $1.33(1.14-1.56)$ & $<0.001$ & $1.06(0.89-1.26)$ & 0.525 \\
\hline
\end{tabular}

Participants who died of COVID-19 had a median age at death of 76 years (interquartile range 71-78 years)

$H R$ hazard ratio, $C I$ confidence interval, IRR incidence rate ratio

*Adjusted for age, sex, ethnicity, month of assessment, Townsend deprivation quintile, household income, BMI category, smoking status, diabetes, systolic blood pressure, diastolic blood pressure, self-reported health rating, and long-standing illness, disability or infirmity

In the current analysis, we therefore linked baseline serum 25(OH)D concentration in 341,484 UK Biobank participants with complete data on covariates to Death Register data. In the sample, 203 participants died due to COVID-19 infection. Deaths occurred between the 5th of March and 25th of April 2020. We explored whether serum 25(OH)D concentration as a continuous measurement, or vitamin $\mathrm{D}$ deficiency or insufficiency (defined as serum $25(\mathrm{OH}) \mathrm{D}<25$ and $<50 \mathrm{nmol} / \mathrm{L}$, respectively), were associated with risk of COVID-19 death using Cox proportional hazards regression analysis.

Univariably, several covariates were associated with COVID-19 mortality and infection (Table 1). Notably, black and South Asian ethnicity, obesity, and lower socioeconomic status are also associated with lower 25(OH)D concentration.

The COVID-19 mortality results followed the same pattern that we previously observed for COVID-19 infection [7]. Lower 25(OH)D concentration and vitamin D deficiency were both associated with higher risk of COVID19 death univariably, but not after adjustment for potential confounders (Table 2). Multivariate models were adjusted for all measured confounders as detailed in the table legend. Vitamin D insufficiency was not associated with risk of COVID-19 death univariably or multivariably. Furthermore, we repeated our previous analysis of the association between 25(OH)D and confirmed COVID-19 infection with additional cases, using univariable and multivariable poisson regression of inpatient diagnosed infection. There were 656 confirmed inpatient COVID-19 cases. Again, 25(OH) $\mathrm{D}$ concentration and vitamin D deficiency were associated with COVID-19 infection univariably but not multivariably (Table 2).

The variables significantly associated with risk of COVID-19 mortality in multivariate analysis were age (HR 1.12; 95\% CI 1.10-1.15; $p<0.001$ per year), male sex (HR 2.12; 95\% CI 1.56-2.89; $p<0.001$ ), black ethnicity
(HR 8.13; 95\% CI 4.56-14.50; $p<0.001$ ), obesity (HR 1.68; 95\% CI 1.11-2.56; $p=0.015$ compared with normal weight), socioeconomic deprivation (highest Townsend deprivation quintile compared with lowest HR 1.96; 95\% CI 1.24-3.09; $p=0.004$ ), and diabetes (HR 1.96; 95\% CI 1.34-2.86; $p=0.001)$. These findings are consistent with other studies, lending strong external validity.

The main limitation of using UK Biobank for this analysis is the $\sim 10$ year time period between baseline $25(\mathrm{OH}) \mathrm{D}$ measurement and COVID-19 infection. We examined the concordance rates of vitamin D deficiency in a subsample of 15,473 participants who had measurements taken both at baseline and at a follow-up visit (on average 4.3 years later). Concordance in this group was $84 \%$.

If there is a causal link, vitamin D supplements would present an appealingly cheap low risk intervention. However, currently there is no evidence that supplements will reduce risk of COVID-19 infection [4], or acute respiratory tract infections more generally [6]. NHS guidelines already recommend that all UK residents take vitamin D supplements in the winter, and furthermore that certain groups who are more likely to be deficient (for example those with darker skin) take them throughout the year [9]. We await the results of randomised controlled trials to determine whether there should be any change to these guidelines and consequently clinical practice. For now, recommendations for vitamin D supplementation to lessen COVID-19 risks appear premature and, although they may cause little harm, they could provide false reassurance leading to changes in behaviour that increase risk of infections.

Funding CEH is funded by HDR-UK (ref Edin-1). NS acknowledges support from the British Heart Foundation Research Excellence Award (RE/18/6/34217). 
Code availability All analyses were undertaken using Stata v14.

Availability of data and material Data can be accessed via the procedures detailed in the UK Biobank website (https://www.ukbiobank. ac.uk/).

\section{Compliance with ethical standards}

Conflict of interest The authors declare that they have no conflict of interest.

Ethics approval UK Biobank received ethical approval from the North West Multi-Centre Research Ethics Committee (REC reference: 16/ NW/0274) and was conducted in accordance with the principles of the Declaration of Helsinki.

Informed consent All participants gave written informed consent for data collection, analysis, and record linkage. All participants gave written informed consent for publication of research.

Open Access This article is licensed under a Creative Commons Attribution 4.0 International License, which permits use, sharing, adaptation, distribution and reproduction in any medium or format, as long as you give appropriate credit to the original author(s) and the source, provide a link to the Creative Commons licence, and indicate if changes were made. The images or other third party material in this article are included in the article's Creative Commons licence, unless indicated otherwise in a credit line to the material. If material is not included in the article's Creative Commons licence and your intended use is not permitted by statutory regulation or exceeds the permitted use, you will need to obtain permission directly from the copyright holder. To view a copy of this licence, visit http://creativecommons.org/licenses/by/4.0/.
2. Sattar N, Welsh P, Panarelli M, Forouhi NG (2012) Increasing requests for vitamin $\mathrm{D}$ measurement: costly, confusing, and without credibility. Lancet 379:95-96. https://doi.org/10.1016/S0140 -6736(11)61816-3

3. The Centre for Evidence-Based Medicine. (2020) Vitamin D: a rapid review of the evidence for treatment or prevention in COVID-19. https://www.cebm.net/covid-19/vitamin-d-a-rapid -review-of-the-evidence-for-treatment-or-prevention-in-covid -19/. Accessed 26 Jun 2020

4. NICE. COVID-19 rapid evidence summary:vitamin D for COVID19. https://www.nice.org.uk/advice/es28/evidence. Accessed 11 Aug 2020.

5. (2020) Welsh Government. COVID-19 workforce risk assessment tool. https://gov.wales/checkyourrisk. Accessed 11 Aug 2020

6. Scientific Advisory Committee on Nutrition. Vitamin D and acute respiratory tract infections. https://app.box.com/s/g0ldpth1up fd7fw763ew3aqa3c0pyvky. Accessed 11 Aug 2020.

7. Hastie CE, Mackay DF, Ho F et al (2020) Vitamin D concentrations and COVID-19 infection in UK Biobank. Diabetes Metab Syndr Clin Res Rev 14:561-565. https://doi.org/10.1016/j. dsx.2020.04.050

8. Raisi-Estabragh Z, McCracken C, Bethell MS et al (2020) Greater risk of severe COVID-19 in Black, Asian and Minority Ethnic populations is not explained by cardiometabolic, socioeconomic or behavioural factors, or by $25(\mathrm{OH})$-vitamin D status: study of 1326 cases from the UK Biobank. J Public Heal. https://doi. org/10.1093/pubmed/fdaa095

9. NHS. Vitamins and minerals: Vitamin D. https://www.nhs.uk/ conditions/vitamins-and-minerals/vitamin-d/. Accessed 26 Jun 2020

\section{References}

1. Mitchell F (2020) Vitamin-D and COVID-19: do deficient risk a poorer outcome? Lancet Diabetes Endocrinol 8:570. https://doi. org/10.1016/S2213-8587(20)30183-2 